\title{
AC 2010-213: DEVELOPING MICROFABRICATION CAPABILITIES ACROSS THE AMERICAS: A CASE STUDY
}

\section{Maria Jose Yanez, Universidad de Santiago de Chile}

Maria Jose Yanez is a fifth year undergraduate student in the Departamento de Fisica at Universidad de Santiago de Chile

\section{Ernesto Gramsch, Universidad de Santiago de Chile}

Dr. Ernesto Gramsch is Profesor de Fisica en Departmento de Fisica at Universidad de Santiago de Chile

\section{Roberto Santander, Universidad de Santiago de Chile}

Dr. Santander is Profesor de Ingenieria Mecanica at Universidad de Santiago de Chile

\section{Cecilia Richards, Washington State University}

Dr. Cecilia Richards is Professor of Mechanical Engineering at Washington State University

\section{Robert Richards, Washington State University}

Dr. Robert Richards is Professor of Mechanical Engineering at Washington State University 


\title{
Developing Microfabrication Capabilities across the Americas: A Chilean - US Case Study
}

\begin{abstract}
This paper describes collaborative work between Chilean and American universities to develop microfabrication capabilities to educate Chilean students in design, development and fabrication of Microelectromechanical Systems (MEMS). Microfabrication facilities are extremely rare in Latin American universities, and students there have virtually no exposure to this key technology. To our knowledge, the facility documented in this paper is the first microfabrication facility to be developed for university education in Chile and only the second in Latin America. Challenges included the extremely small budget available for the project and the paucity of educational and resource materials available in Latin America.

\section{Introduction}

Microfabrication is a key enabling manufacturing technology. Almost all digital electronics are manufactured using these microfabrication techniques. Over the last thirty years, the suite of available microfabrication techniques has expanded dramatically. This expansion has enabled the rapid rise of a new field, Microelectromechanical Systems or MEMS. The economic impact of these manufacturing methods is enormous. For this reason, many facilities and programs have been developed in educational institutions in the US to introduce students to microfabrication methods [1-5]. Educating both undergraduate and graduate students in microfabrication and batch manufacturing methods is commonly seen as key to promoting US economic competitiveness, by contributing to the talent base required by industry and by enhancing public understanding of one of the key technologies shaping the industrial world.

While many microfabrication facilities can be found in US colleges and universities, information about such facilities in educational institutions in Latin American is sparse. It appears that extremely few such facilities exist and that very few Latin American students have the opportunity to learn microfabrication methods first hand, or to gain hands-on experience actually fabricating microdevices. Thus while products such as cell phones, personal computers, and video games are ubiquitous in Latin America, experience with the micromanufacturing techniques used to produce these consumer items is extremely rare. To our knowledge, only one university in Spanish-speaking South America has a cleanroom dedicated to education and research, the Universidad de Los Andes in Colombia [6, 7]. Certainly, when we began our project there was no university-based microfabrication facility in Chile.
\end{abstract}

For this reason, we decided to develop facilities in Chile to enable students there to learn basic surface micromachining techniques sufficient to fabricate simple MEMS structures. Our goal then was to develop a university-based microfabrication capability to provide opportunity for local students to gain experience in micromachining techniques. In addition, since Chilean students are strongly motivated by national need and national 
pride, it was deemed important to develop a microfabrication capability focused on addressing a significant national priority. The importance of the copper industry to the Chilean economy, led us to focus on micromachining techniques that could be used to fabricate MEMS sensors suitable for use in the Chilean copper industry.

To develop the kind educational facility that would enable Latin American students to gain hands-on experience in MEMS technology, our Chilean-US team needed to address multiple needs. Our first and most daunting challenge was to develop the infrastructure required for microfabrication processes. In particular, microfabrication requires a clean and safely ventilated environment in which to work. Second, we needed a mechanism to begin educating Chilean students in microfabrication protocols and techniques. Third, we needed to acquire and or construct several key pieces of microfabrication equipment. Fourth, we needed to source microfabrication consumable supplies such as photoresist and etchants. Meeting each of these needs has had its challenges.

First, since microfabrication processes require a well-controlled clean space in which to operate, we have designed, built and tested a four square meter, class 3000 clean room. Second, to begin the education of Chilean students in microfabrication techniques, we have begun hosting Chilean students at our US based microfabrication facility. A onemonth visit by our first student in September, 2009 initiated this exchange and enabled us to develop appropriate fabrication protocols suitable for implementation at the new Chilean microfabrication facility. This first student has already begun fabrication of key pieces of MEMS-based heat flux sensors, that are both suitable for use in the Chilean copper industry and amenable to micromanufacture using only relatively low-cost surface micromaching techniques.

The last two challenges are works in progress. While we have worked to implement our microfabrication processes using readily accessible laboratory equipment, several pieces of equipment are still needed. The notable exceptions have been the design of a spin coater (for deposition of photoresist), and a mask aligner (for exposure of the photoresist during photolithography), amenable to construction Chile. Finally, much effort has gone into finding sources of processing supplies for our Chilean operation, in particular sources for photoresist and developer. This paper describes the results of our efforts to overcome these four challenges.

\section{Approach}

In order to educate Chilean student in MEMS fabrication techniques, we began by developing the capability to expose students to two basic surface micromachining techniques: photolithography, and wet chemical etching. Efforts focused first on constructing required infrastructure, and second on educating students in processing skills.

\section{Development of Microfabrication Infrastructure: Cleanroom Construction}

The infrastructure for microfabrication most conspicuously lacking was a clean environment in which to carry out fabrication procedures. No existing cleanroom or 


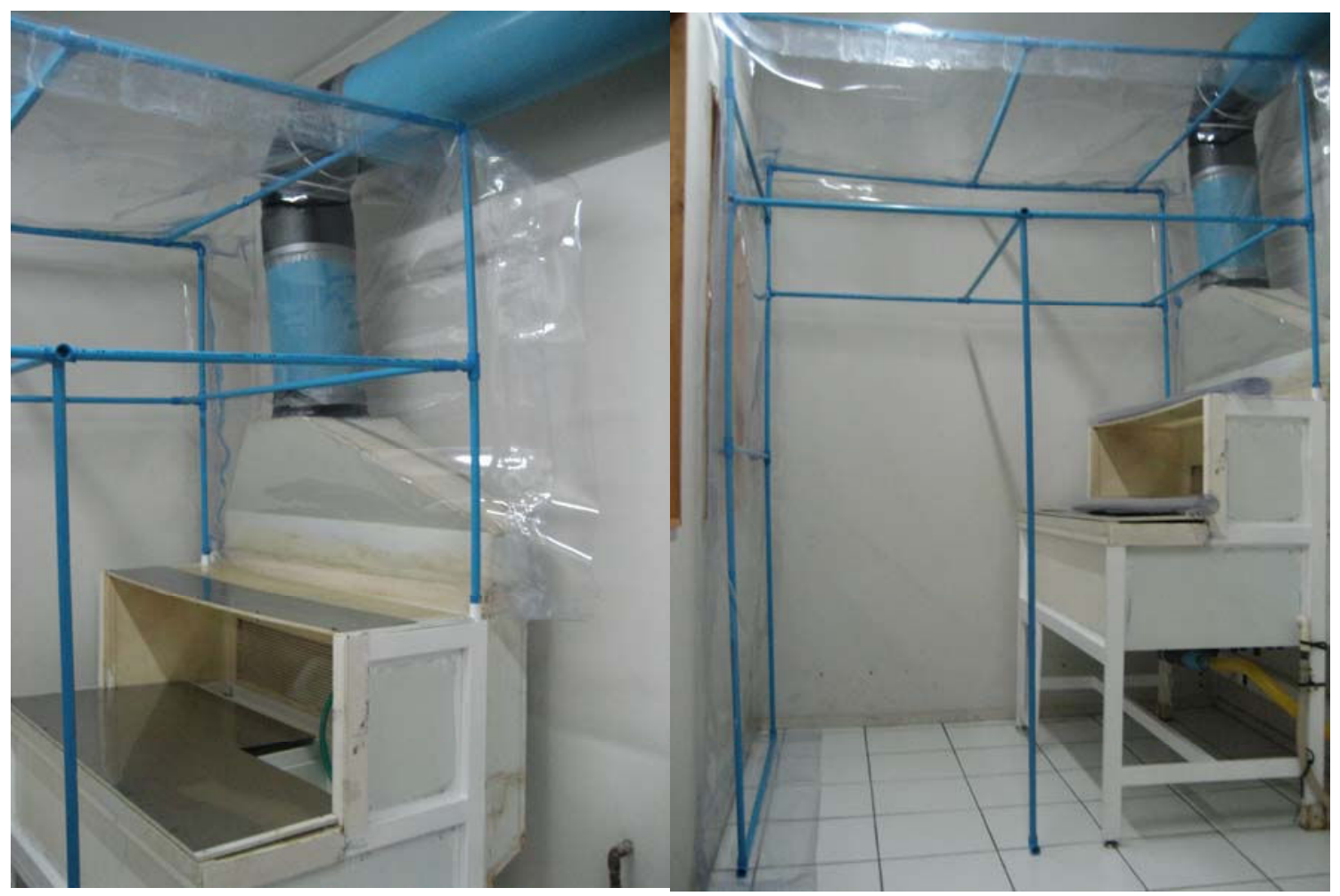

Fig. 1. Two views of the refurbished wet-etch station with exhaust pipe installed. The stainless steel work surface and sink are visible in the photo on the left.

clean hood was available for use by us. Existing lab spaces that were available had measured particle counts of $10^{5}$ particles per cubic foot; too high to permit the kind of photolithographic process we aimed for. As a result, in order to provide sufficiently clean space for fabrication, we needed to build a small cleanroom.

To build the cleanroom, we selected a small underutilized room with electrical power, city water, drainage, and most importantly, access to the exterior through a set of small windows. Fortuitously, the room communicated with the rest of the interior lab space through two large windows, and a set of two doors arranged like an airlock. This circumstance enabled isolation of the cleanroom space from the rest of the lab space for greater cleanliness.

The cleanroom itself was designed to be constructed at lowest cost, by the available labor, consisting of one faculty member, one student and one technician. An old wet-etch station, discarded by a US company and transported to Chile several years ago, formed the core of the cleanroom. The station consisted of a series of sinks, with plumbing for water supply and drainage, a small work surface, and an air exhaust system. The exhaust system was designed to pull air through a grille located on the wall just above and behind the work surface and sinks, through a plenum and then out through a round exhaust duct at the top of the station. Figure 1 shows two views of the wet-etch station. 
This wet-etch station was stripped down, thoroughly cleaned and painted. One sink in the wet etch station, was plumbed to the room floor drain. The remaining sinks were covered over with a new work surface. This new work surface was built of quarter inch (1/4") thick PVC sheet covered with one thirty-second inch (1/32") thick stainless steel sheet in order to be chemically resistant and easily cleanable.

The etch station exhaust system was vented to the outside of the building by epoxying twelve inch diameter PVC pipe to the round exhaust duct at the top of the station. Approximately five meters ran from the etch station to the room's exterior window. Another five meters of PVC pipe ran up the exterior wall of the building to safely vent the exhaust from the etch station away from passersby.

To create a clean, enclosed space around the refurbished wet-etch station a soft-sided cleanroom was built onto it. The cleanroom thus took the form of a tent, $2.35 \mathrm{~m}$ high, 1.8 $\mathrm{m}$ long and $1.35 \mathrm{~m}$ wide, with walls and ceiling constructed of $0.30 \mathrm{~mm}$ thick PVC sheet, supported on a framework of one half inch (1/2") diameter PVC pipe. The foot print of the cleanroom was thus $1.8 \mathrm{~m} \times 1.35 \mathrm{~m}$, or $2.4 \mathrm{~m}^{2}$. The volume of the clean room was only $5.7 \mathrm{~m}^{3}$. With the wet etch station integrated into one of the short $(1.35 \mathrm{~m})$ sides of the soft-sided cleanroom, this footprint allowed sufficient space for two adults to work comfortably side by side. However, provision for storage space within the clean room was minimal.

The PVC framework supporting the ceiling and wall of the cleanroom was built by cutting PVC pipe to length and gluing pipe sections together using standard elbow, tee and straight connectors. The PVC pipe was glued together in the form of a rectangular solid, with some bracing for rigidity. One side of the frame was epoxied directly to the wet etch station. Once the framework was assembled and standing, the soft PVC walls and ceiling were put in place. Both walls and ceiling were constructed of $0.30 \mathrm{~mm}$ thick PVC sheets. The PVC was purchased by the meter, in $1.5 \mathrm{~m}$ wide sheets.

A single $1.5 \mathrm{~m}$ sheet was sufficient for the short $(1.35 \mathrm{~m})$ side walls and for the ceiling. Two sheets glued side-by-side, with a $10 \mathrm{~cm}$ overlap, were used for the long $(1.8 \mathrm{~m})$ side walls. Corners were constructed by cutting $20 \mathrm{~cm}$ wide strips of PVC sheet, and scoring the strips lengthwise down the middle with knife. The scored line was then creased to form the corner, and the two adjacent wall sections were glued onto the $10 \mathrm{~cm}$ wide flaps on either side of the corner. Wall sections were glued to the ceiling at the top of the structure, and folded under the PVC pipe framework at the bottom. The bottom flaps of the wall were then taped directly to the floor. Likewise wall flaps on the side of the cleanroom at the wet etch station were taped to the station. Figure 2 shows the wet-etch station inside the cleanroom, as seen through the transparent PVC walls. The PVC pipe framework is clearly visible in the photograph.

An airlock entry was created by building an anteroom of PVC pipe framework and PVC sheet, $2.35 \mathrm{~m}$ high, $0.95 \mathrm{~m}$ wide and $1.2 \mathrm{~m}$ long adjacent to one long side of the cleanroom. One entered the cleanroom, by first entering into the anteroom using a vertical two-meter long zipper sewn into the short side $(0.95 \mathrm{~m})$ wall of the anteroom. 


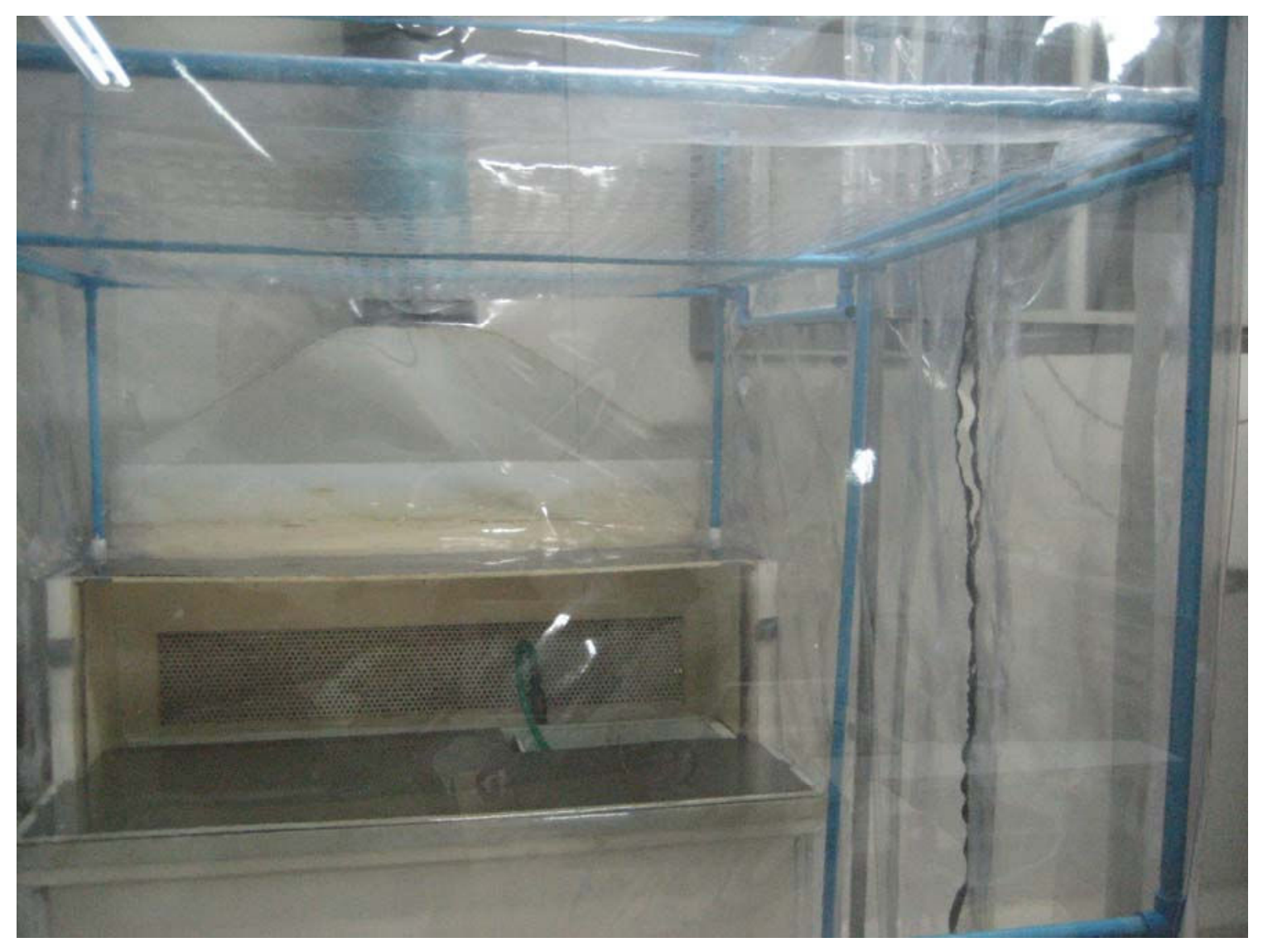

Fig. 2. The wet-etch station as seen through the transparent PVC walls of the cleanroom. The zipper entryway is visible on the right side of the photograph.

One then passed from the anteroom to the cleanroom through a second vertical two-meter long zipper sewn into wall of the cleanroom. The anteroom served not only as an airlock entrance but as a clean environment to enrobe in and disrobe from cleanroom garb, and to store that garb when not in use. Figure 3 shows the anteroom with zippered entrances.

The result of this construction effort was a relatively airtight soft structure, with an airlock entry, room for two workers, a small stainless steel working surface, a sink and provision for air exhaust behind the working surface. A supply of clean air was provided by attaching an air handling unit, fabricated in-house, to the top of the long $(1.8 \mathrm{~m})$ side wall adjacent to the anteroom.

The home-built air handling unit consisted of a centrifugal fan, powered by a one-third (1/3) hp motor (220V, 2.4 Amp), bolted onto a filter box constructed of sheet steel. The centrifugal fan was acquired second hand from a local HVAC used parts store. The filter box, $62 \mathrm{~cm}$ high by $62 \mathrm{~cm}$ wide by $75 \mathrm{~cm}$ long, was designed to accommodate three commercially available filters: (1) a prefilter, (2) a bag filter, and (3) a HEPA filter, in that order. The three filters were secured in place with set screws. Rubber gaskets were 


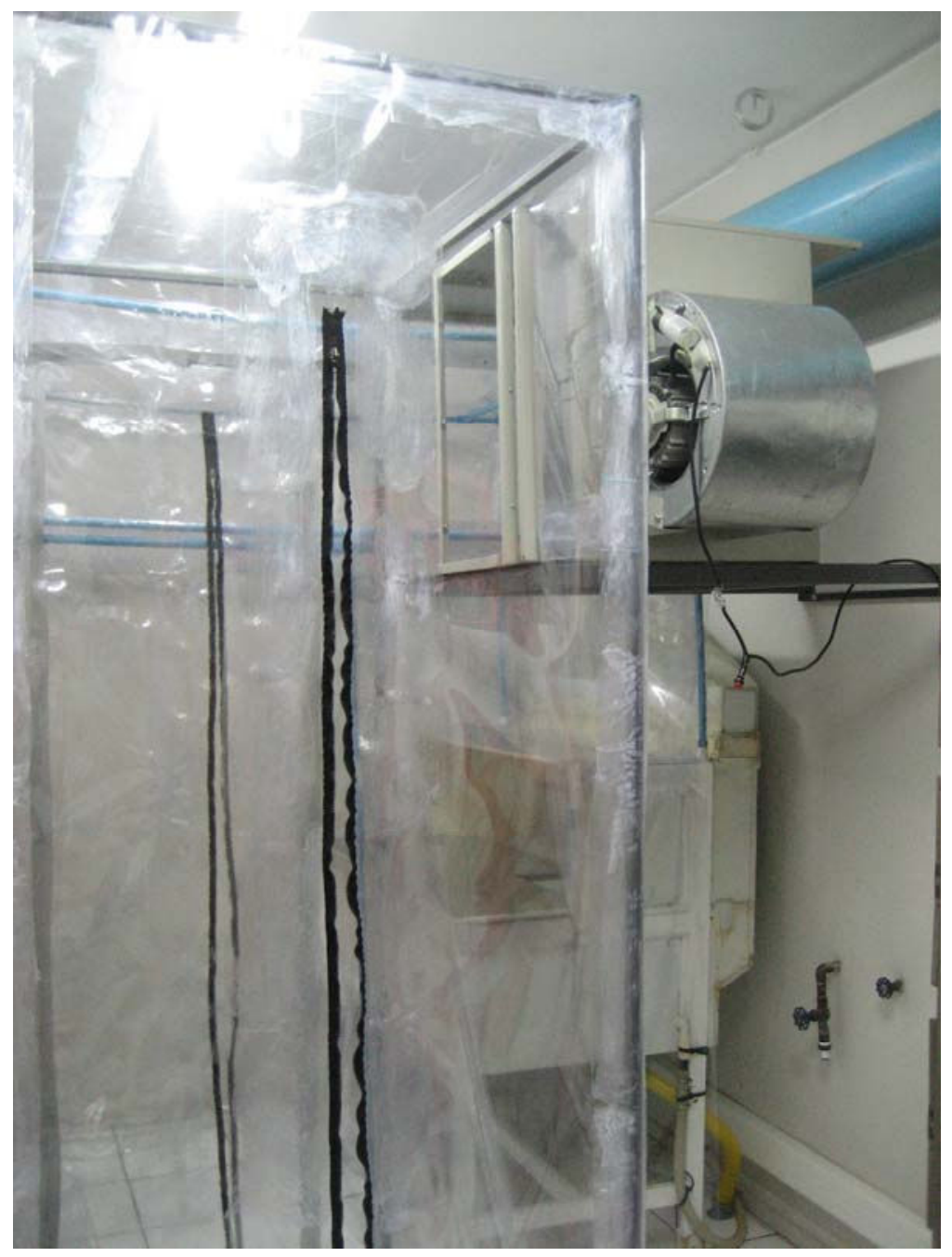

used to ensure an airtight fit for each filter in the filter box. Figure 4 illustrates the air handling unit.

When the air handling unit (centrifugal fan and filter box) was attached to the clean room, and powered up, the centrifugal fan drew air from the surrounding room, and then pushed it through the prefilter, bag filter and HEPA filter. Filtered air then enter into the top of the cleanroom near the ceiling. In this way, the air handling unit pressurized the cleanroom space with filtered air, causing the soft sides of the room to bulge out. A plenum at the top of the cleanroom, created by a PVC sheet through which a grid of one inch holes had been punched, helped distribute the airflow and

Fig. 3. The anteroom to the cleanroom with zippered entrance.

direct it down from the ceiling to the floor of the cleanroom. Air exited the cleanroom through the etch station exhaust system, flowing through the grille behind the work space, and then through the twelve inch (12") PVC exhaust pipe out of the building. In sum, the end result was an open loop ventilation system, with air entering the system at the centrifugal fan, being filtered in the filter box, passing from the ceiling to the floor of the clean space and then exiting through the etch station and exhaust pipe.

The use of such an open-loop ventilation system incurred definite trade-offs. On the one hand the design and construction of our once-through system was dramatically simplified in comparison with a closed-loop system. Likewise, the cost of the open-loop system was much lower than the cost of a closed-loop system. In addition, it was felt that a oncethrough system was safer for the clean room users, since there was no possibility of fumes or vapors being recycled into the workspace. On the other hand, an open-loop system exposed the filters in the air handling unit to much dirtier air than a closed-loop system would have. As a result, the working life of the filters was expected to be much 


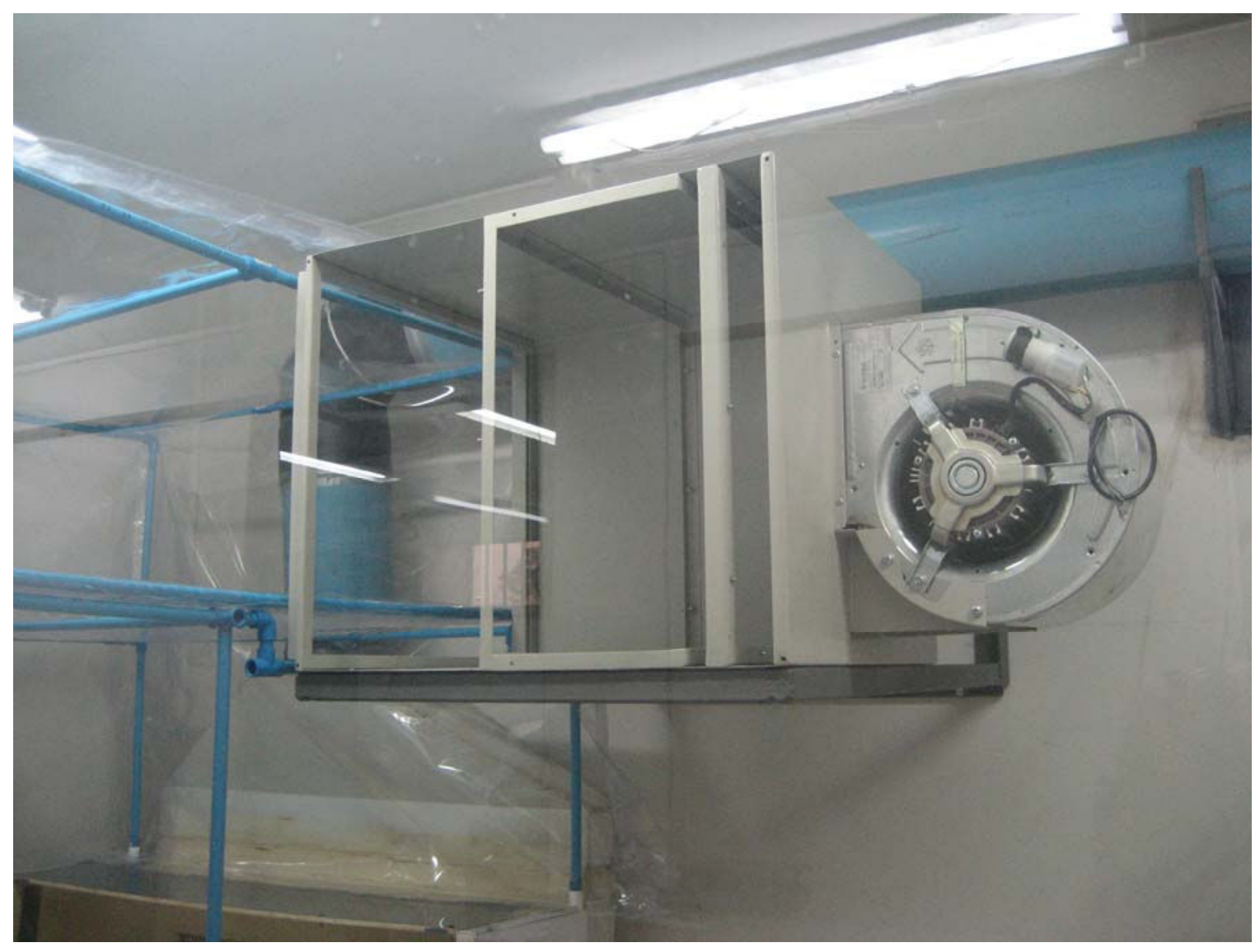

Fig. 4. The air handling unit. The filter box cover is removed, to show the slots where the prefilter, bag filter and HEPA filter are installed. Air flow into the cleanroom is from right to left. The cleanroom exhaust is visible behind the air handling unit.

shorter in our simple once-through ventilation system, than it would have been in a more sophisticated air recycling system. In essence, we traded off a lower first cost of construction of the ventilation system, for a higher operating cost in filter replacements. Given the extremely restrictive budget we worked with, these costs were a significant consideration.

Operation of the cleanroom was very simple. Once, power to the centrifugal fan was turned on, clean filtered air was supplied to the clean space. Using a calibrated hot wire to measure air velocity profiles over the duct between the filters and clean room we determine the measured volumetric flow rate into the cleanroom to be $0.5 \mathrm{~m}^{3} / \mathrm{s}$. Given the small, $5.7 \mathrm{~m}^{3}$, total volume of the clean space, this air flow rate implied an air exchange rate for the cleanroom of one complete air change about every 11 seconds, or just over 300 air changes per hour.

Airborne particle counts were made inside and outside of the cleanroom using a calibrated particulate counter as shown in Fig. 5. Measurements were made just before and during operation of the ventilation system. Before the air handling unit was turned 


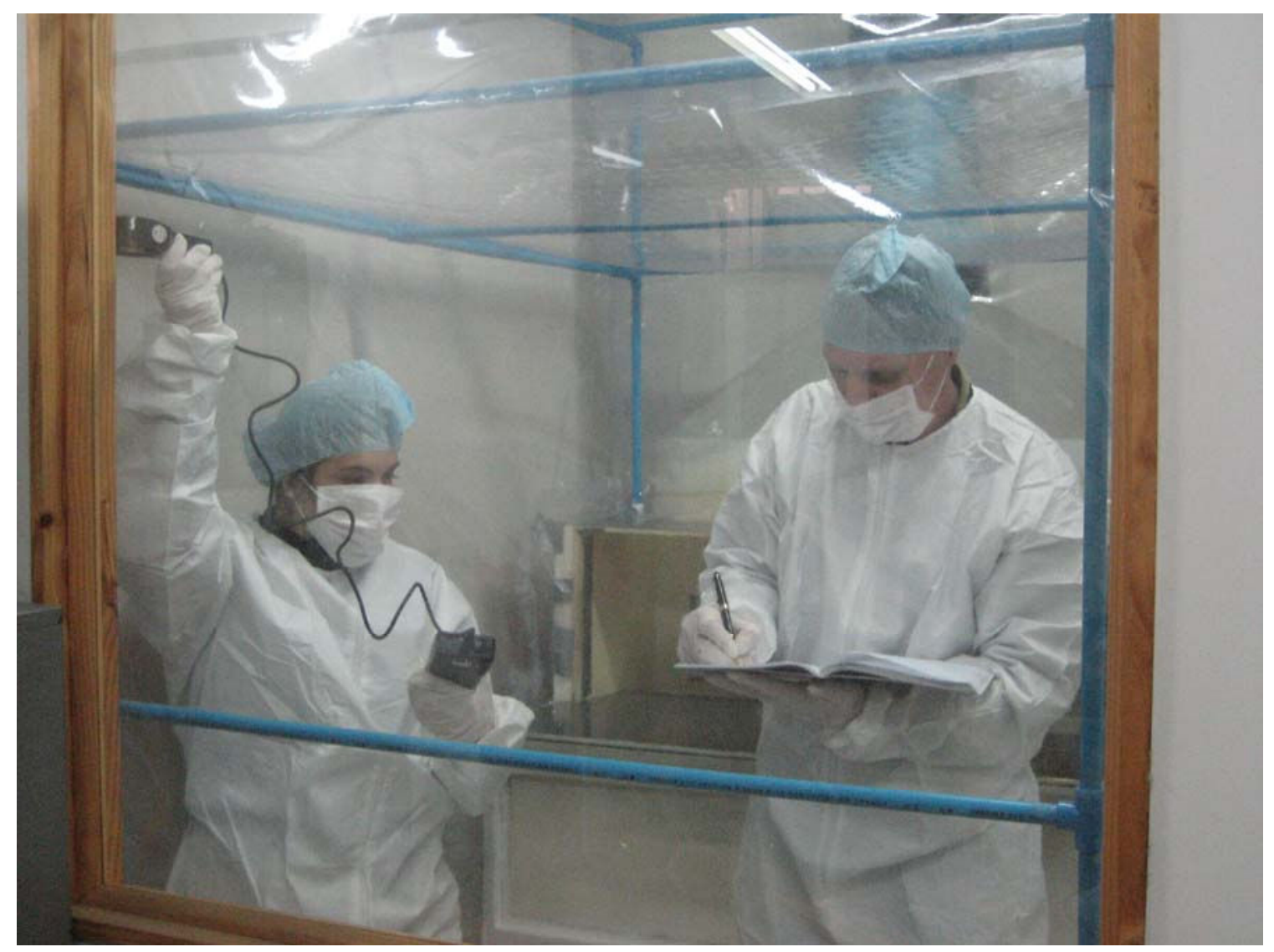

Fig. 5. Measuring particle counts in the cleanroom.

on, measurements of particle counts in the cleanroom indicated the presence of $4 \times 10^{5}$ particles greater than 0.5 micron and $4 \times 10^{3}$ particles greater than 5.0 micron. After ninety minutes of operation, particle counts in the cleanroom dropped down between $2 \times 10^{3}$ and $4 \times 10^{3}$ for particles greater than 0.5 micron and 0 particles greater than 5.0 micron. Particle counts were lowest near the etch station workspace. Outside of the cleanroom particle counts remained constant at $3 \times 10^{5}$ particles greater than 0.5 micron and $3 \times 10^{3}$ particles greater than 5.0 micron. These measurements indicated that with careful use our facility could be operated successfully as a class 3000 cleanroom.

It is instructive to look at the costs incurred in constructing this small facility. Table 1 gives a breakdown of the costs (assuming the then current exchange of 560 Chilean Pesos per US Dollar). The total cost of the cleanroom, excluding labor, is seen to come in at just under 900,000 Chilean Pesos or 1600 US Dollars. The cost of the ventilation system (at 791,000 Chilean Pesos or 1424 US Dollars) is seen to account for $90 \%$ of the cost of the cleanroom. The cost of the cleanroom structure itself is quite small, accounting for less than 100,000 Chilean Pesos or 160 US Dollars. This type of soft-sided cleanroom structure is then extremely economical. In addition, since the structure is easily constructed in a short time by students and/or faculty with minimal experience, it lends itself to organizations with few resources. 


\begin{tabular}{|l|c|c|}
\hline Item & Cost (Chilean Pesos) & Cost (US Dollars) \\
\hline Centrifugal Fan & 180,000 & 324 \\
\hline Stainless Steel Sheet & 18,000 & 32 \\
\hline Filters & 186,000 & 335 \\
\hline Filter Box & 180,000 & 324 \\
\hline PVC Sheet (0.30 mm thick) & 50,000 & 90 \\
\hline PVC Tube (1/2 in. dia.) & 8,800 & 16 \\
\hline PVC Exhaust Tube (12 in. dia.) & 245,000 & 441 \\
\hline Glue, Epoxy, Tape, Zippers & 20,000 & 36 \\
\hline Total Cost & $\mathbf{8 8 7 , 8 0 0}$ & $\mathbf{1 5 9 8}$ \\
\hline
\end{tabular}

Table 1. Cleanroom construction costs.

Educating Chilean Students: Basic Microfabrication Skills

With the cleanroom constructed we turned our efforts toward educating the first Chilean students in microfabrication skills. To this end, a Chilean student traveled to our US university, where she trained for one month in our cleanroom. There, the student learned basic cleanroom skills as well as receiving more specific instruction in metallization techniques, photolithography, and wet chemical etching. The student's training culminated in the fabrication of a set of microfabricated heat flux sensor die. Heat flux sensors were chosen as the first target application for Chilean students because of the importance of the copper industry to that country.

The copper industry is a large part of the Chilean economy; copper production accounts for one third of all government revenue [8]. For this reason, improving the efficiency and productivity of the copper industry is a major national goal. After copper ore has been smelted to form copper matte (typically a mixture of $\mathrm{Cu}_{2} \mathrm{~S}$ and $\mathrm{FeS}$ ), the matte is converted to blister $(98 \%$ pure $\mathrm{Cu})$ by processing it at high temperature $\left(>1000^{\circ} \mathrm{C}\right)$ by blowing air through the hot matte in a Pierce Smith Converter. Infiltration of the molten contents of the converter into cracks in the refractory walls of the converters causes degradation in those walls and is the limiting factor for the lifetime of the converters. Since smelter downtime is very expensive in these large batch operations, predicting the progress of melt infiltration into the walls, the resulting extent of refractory wall degradation and the time to converter failure is of great importance to the industry. As a result monitoring the integrity of the converter walls is a high priority. Melt infiltration into the refractory walls can determined from measurements of wall heat flux, since the melt in the converter is composed largely of high thermal conductivity copper. Robust, accurate and inexpensive heat flux sensors that could be distributed through the walls of a Pierce Smith Copper Converter thus could provide a potential means to monitor the integrity of the converter walls in real time, and more accurately determine remaining wall lifetime $[9,10]$.

MEMS-based heat flux sensors offer one possible way to meet this need. As a result our two groups in Chile and the US decided to develop the capability to fabricate, test and implement MEMS heat flux sensors for the Chilean pyrometallurgical industry. The heat 


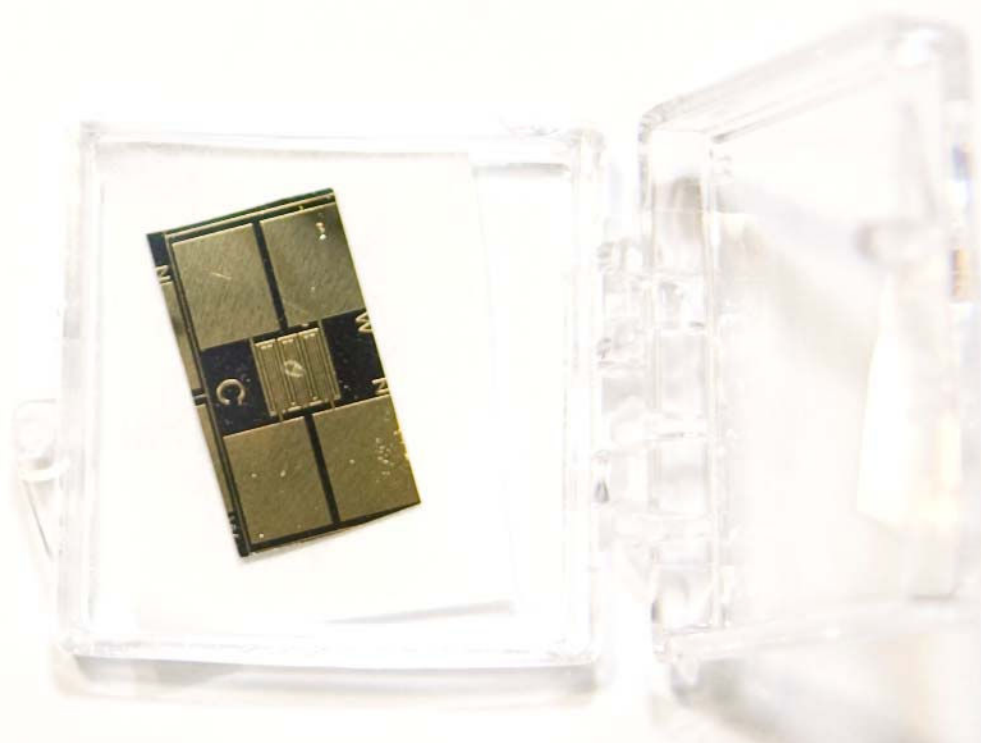

Fig. 6. A surface micromachined die containing a thin-film heater and a resistance thermometer for the MEMS heat flux sensors.

flux sensors we decided to fabricate take the form of a five layer sandwich. They consist of three identical Si die separated by two insulating layers. The three identical Si die each having a thin-film resistance heater and resistance thermometer (RTD) surface micromachined on them. The two insulating layers consist of Kapton sheets, with thickness that may range from 10 microns to 1 millimeter. A heat flux sensor is assembled by stacking the three Si dies and two Kapton sheets alternately. The stack then runs: (1) Si die, (2) Kapton sheet, (3) Si die, (4) Kapton sheet, (5) Si die.

The sensors are used to determine heat flux by measuring the temperature difference across the known thermal resistance of the insulating Kapton layers, using the RTD's micromachined on the Si die. The heat flux sensors can be calibrated in situ by dissipating electrical power at a known rate in one of the thin film heaters, and measuring the resulting temperature gradients between the RTD's on each of the three Si die. This approach, leads to a very robust heat flux sensor, whose calibration can be periodically validated while under operating conditions, a significant advantage for sensors operating under the demanding conditions of a Pierce-Smith Converter.

This kind of sensor offers an excellent target application for students learning microfabrication processes. The heat flux sensor die are fabricated using three inch, single-surface polished silicon wafers as the substrate. First, a two-micron oxide coat is thermally grown on the wafer. The wafer surface is then metalized by sputtering on a 300 


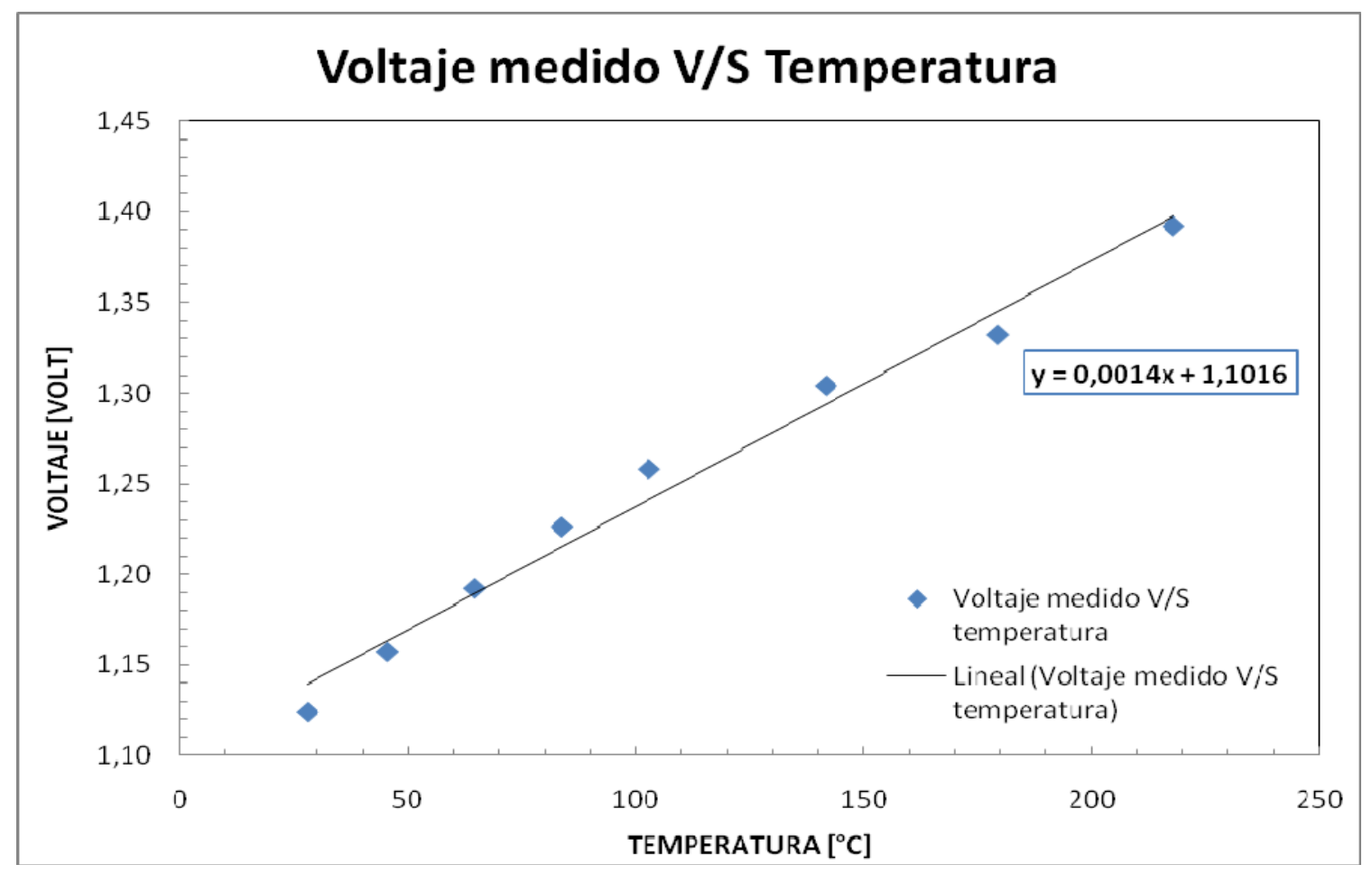

Fig. 7. A sample calibration curve for a resistance thermometer on one of the surface micromachined device die.

$\mathrm{nm}$ layer of $\mathrm{Au}$. Positive photoresist is spun onto the wafer and then exposed using a mask aligner to define the geometry of the thin film heater and resistance thermometer. The photomask is created by drawing the heater and resistance thermometer geometry using Corel Draw and then printing the design on a plastic transparency using a high resolution laser printer. This approach results in a mask with a dot size of $\sim 25$ microns, sufficient resolution for our devices with no details smaller than $\sim 50$ microns. Upon exposure, the photoresist is developed, and the Au layer wet etched to reveal the device geometry. Each three-inch wafer can be diced to produce sixteen devices. All procedures were accomplished by our first Chilean MEMS student. All procedures, with the single exception of the metallization, can be realized in the new Chilean microfabrication facility. Figure 6 shows a sample device die fabricated by a Chilean student.

Upon returning to her home university in Chile, our first student began calibrating the device die. Figure 7 illustrates a sample calibration of one of the resistance thermometers produced. At this point, the device die await assembly into complete heat flux sensors for testing and implementation.

Summary

To date, we have succeeded in constructing and commissioning a $2.4 \mathrm{~m}^{2}$, class 3000 cleanroom in a Chilean university lab, suitable for educating Latin American students in basic microfabrication techniques. This was accomplished on a budget of only 1,600 US dollars. All components for the cleanroom were locally sourced. All labor was provided 
by three personnel: one faculty member, one student and one technician. The first Chilean student has begun learning microfabrication protocols, working in our US microfabrication facility and in the Chilean facility. The first learning project, micromachining heat flux sensor dies has been completed. Those die, transported back to Chile, have been calibrated and now await assembly into finished heat flux sensors. Those MEMS heat flux sensors, designed to illustrate basic surface micromachining techniques, are suitable for use in diagnosing wall degradation in Pierce-Smith copper converters now in use by the Chilean copper industry.

Future work for this ongoing project includes acquiring or building a spin coater (for deposition of photoresist), and a mask aligner (for exposure of the photoresist during photolithography), for use in our Chilean cleanroom. Finally, more effort is required to locate economical sources of processing supplies for our Chilean operation, in particular sources for photoresist and developer.

\section{Bibliography}

1. J. D. Musgraves, B.T. Close, D.M. Tanenbaum, A maskless photolithographic prototyping system using a low-cost consumer projector and a microscope, Am J. Physics, 73 (10) pp 980 - 990, 2005.

2. S.J. Stelick, W.H. Alger, J.S. Laufer, A.M. Waldron, C.A. Batt, Hands on classroom photolithography laboratory module to explore nanotechnology, J. Chem. Ed., 82 (9) 1361 - 1364, 2005.

3. K.L. Berkowski, K.N. Plunkett, Q. Yu, J.S. Moore, Introduction to photolithography: Preparation of microscale polymer silhouettes, J. Chem. Ed., 82 (9) $1365-1369,2005$.

4. A.M. Christenson, G.W. Corder, T.C DeVore, B.H. Augustine, A photolithography laboratory experiment for general chemistry students, J. Chem. Ed., 80 (2) 183 - 186, 2003.

5. P.S. Gworzdz, NSF Microfabrication Workshops, IEEE Transactions on Education, 39 (2) 211-216, 1996.

6. A.G. Avila Bernal, Colombia avanzado en nanotecnologia, Innovacion y Cienca, XII (4) 62-67 2005.

7. A.G. Avila Bernal, Research directions and challenges in nanoelectronics, J. Nanoparticle Res., 8 (6) 841-858 2006.

8. www.cia.gov/library/publications/the-world-factbook/geos/ci.html, CIA - The World Factbook - Chile

9. C. Goni, M.F. Barbes, V. Bazan, E. Brandaleze, R. Parra, L.F.V. Verdeja, The mechanism of thermal spalling in the wear of the Pierce-Smith copper converter, J. Ceramic Soc. Japan, 114 (8) 672-675, 2006.

10. R. Parra, L.F.V. Verdeja, M.F. Barbes, C. Goni, V. Bazan, Analyzing furnacelining integrity using nodal wear modeling, JOM, 29 -36, Oct., 2005 